\title{
A Decision Making Tool for Assessment of Leasing Policies of a Satellite Operator
}

\author{
Elena Sarri, PhD Candidate \\ George P. Papavassilopoulos, Professor \\ Department of Electrical and Computer Engineering, National Technical University of Athens \\ 9 Iroon Polytexneiou Str. GR-157 73 Zografou Athens, Greece \\ e-mail: elena@netmode.ntua.gr
}

\section{KEYWORDS}

Satellite Communications, Leasing Policies, Modelling, Stochastic Optimization.

\begin{abstract}
This paper presents the results of a study concerning the evaluation of different decisions of a Satellite Operator leasing capacity to different customers requesting different services. The study was done for Hellas-Sat (the Greek satellite operator) and addresses a real situation. Data concerning demands of several customers were collected using those available in particular organizations and corresponding to the area that Hellas-Sat covers. These data was first classified and statistically treated. Second a decision tree has been created where the statistical characteristics taken from the first part of the study, were used in order to derive optimal leasing policies/scenarios and characterize them in terms of both profit and risk. The final outcome is a decision making tool which can be used by a Satellite Operator in order to evaluate possible states, profits and associated risks.
\end{abstract}

\section{INTRODUCTION}

The continuous growth of the satellite communications market and its applications nowadays, make the need of a decision making tool for a satellite operator obvious. A satellite provider offers a variety of services to users from different market segments. Each service has different bandwidth demand and therefore a different price. Each customer has different characteristics (time of lease, bandwidth). We present a model that compares different scenarios/cases of combinations of customers that want to hire of satellite capacity.

Each scenario leads to a decision tree with main goal the maximization of the profits for the satellite provider. This maximization problem is described in terms of real and expected revenues, along with the corresponding probability of getting them. Mean values and standard deviations of the portions involved have been calculated and used to asset risk.

The aim of the provider is to maximize profit. This is an optimization problem that involves actual problems that any satellite operator faces. The ability of real time decisions has a significant effect to the viability and profitability of the company in the growing and competitive satellite market. The projected decision making model is an applied tool for managerial purposes which deals with real problems.

A mathematical model was created that takes into account the uncertainty and hence the risk. The model includes the recording of possible states. Accordingly their quantification and analysis take place, considering the possible benefits but also the resulting risk. Depending on these data different alternative enterprising steps that the company might follow will result.

Apart from the determination of uncertainty the model incorporates the possibility of time evolution. The decisions are taken successively in a time horizon and consequently the model is dynamic, taking into account the evolution in time. This characteristic involves the analysis of the risk of a decision in a competitive environment. This fact is compatible with the studied application of a satellite provider since it operates in a aggressive and growing market. The international developments of the satellite market have been taken into account since pricing data for different services and thus different capacity demand have been gathered. These data have been statistically treated and produced mean values and standard deviations for each service and each bandwidth demand when needed (a lot of these data where provided to us by Hellas-Sat). For the mathematical background of this work see (Bertsekas 2005 and 2001) and (Ross 1983). Related theoretical work available in the open literature is not very common due to the relatively recent appearance of the satellite operator business, but nonetheless see (Guikema and Pate'-Cornell) for related work.

\section{STAGES OF WORK}

The first stage of this study involved the recording and the evaluation of the pricing data coming from the international market of leasing satellite capacity as well as their statistical treatment.

The goal of the proposed model, implemented in the second stage, is to enable the decision maker to determine the best possible scenario for the satellite operator, which is the scenario with the larger amount of income.

The hypothesis that a satellite operator has different lease demands from different customers is made. Each customer wants to hire satellite capacity with certain bandwidth, time of lease and has a certain charge. The outcome of the model gives guides to which combination of them is more profitable for the operator. 
A scenario can be customized by entering the characteristics of the possible customers which are: Service, that is the type of the requested service, $t$ start, that is the month of beginning of lease, $t$ end that is the month of end of lease and produces the requested duration of lease with respect to the time evolution, $w(\mathrm{MHz})$, that is the requested bandwidth, and $C$ (Euros) that is the corresponding cost of lease. In the structure of the model, the possibility of beginning the hire in different time periods (different months) has been included. Table 1 shows an example of the input parameters of the model.

Table 1: Input Parameters

\begin{tabular}{|c|c|c|c|c|c|}
\hline $\begin{array}{c}\text { Accession } \\
\text { number of } \\
\text { customer }\end{array}$ & $\begin{array}{c}\text { Type of } \\
\text { Service }\end{array}$ & $\begin{array}{c}\mathrm{t} \text { start } \\
\text { (month) }\end{array}$ & $\begin{array}{c}\mathrm{t} \text { end } \\
\text { (month) }\end{array}$ & $\begin{array}{c}\text { Duration } \\
\text { of lease } \\
\text { (months) }\end{array}$ & $\begin{array}{c}\text { Requested } \\
\text { w (MHz) }\end{array}$ \\
\hline \hline $\mathbf{1}$ & A & 1 & 15 & 15 & 15 \\
\hline $\mathbf{2}$ & B & 3 & 10 & 8 & 20 \\
\hline $\mathbf{3}$ & C & 2 & 20 & 19 & 1 \\
\hline $\mathbf{4}$ & D & 2 & 15 & 14 & 5 \\
\hline $\mathbf{5}$ & E & 3 & 10 & 8 & 30 \\
\hline $\mathbf{6}$ & F & 11 & 15 & 5 & 20 \\
\hline
\end{tabular}

The indicative cost for each service, was calculated using the data collected from the global satellite market. From the gathered data there was a classification of the possible services that a satellite operator could offer. These services correspond to different bandwidth demands and are presented in Table 2 . The statistical analysis included the pricing for the equivalent capacity per $36 \mathrm{MHz}$ per month and the duration of lease in months. In each service the mean value the standard deviation and the percentage of difference between standard deviation and mean value of these sizes was determined as well as the crosscorrelation of them. Similarly, we did the calculations for the duration of hire.

Table 2: Types of Services

\begin{tabular}{|c|}
\hline Services \\
\hline VSAT \\
\hline Telephony \\
\hline IP Gateway \\
\hline Corporate \\
\hline Broadcast \\
\hline Video Contribution \\
\hline Media company \\
\hline Government \\
\hline
\end{tabular}

\section{CONCEPT OF THE MODEL}

The model does the comparison of 6 at most customers with maximum asked time of lease 60 months, a fact that is considered to be a normal rate of customers in a certain period and easily can be expanded to more customers wanting satellite capacity for more than 2 years. All the possible combinations of customers are calculated (see Figure 1 Group A) where a description of these combinations is taking place in terms of possibility to implement or not. Specifically there is an indication of Possible, Not Possible or Negotiable Combination in each combination of customers.

A Negotiable Combination is the combination of customers that exceeds by $1 \mathrm{MHz}$ at most the highest possible capacity that a transponder can serve i.e. 36 $\mathrm{MHz}$, which can probably constitute an issue of negotiation between the provider and the consumer. A Possible Combination is the feasible combination of customers from the point of view of the maximum capacity of the transponder and a Not Possible Combination is a not feasible one. So the proposed tool gives the benefit of shorting by the Description of combination so as to present all the Possible combinations (see Figure 1 Group B). The next step is to decide which of these possible combinations are the best to compare by examining the most promising ones. This is done by selecting the combinations with the highest amount of total occupied capacity, which is the sum of the requested capacity of each customer of the combination. Obviously the more bandwidth is occupied from a transponder the more profit will be for the firm. These are the 'Real Revenues' (see Figure 1 Group C).

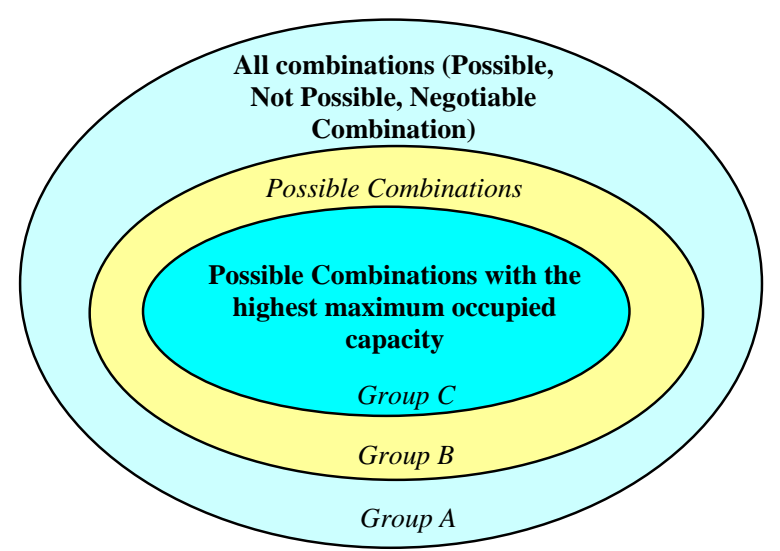

Figure 1: Possible Combinations of Customers

The next step is to decide which of these combinations with high occupied capacity is the more profitable. The criteria that can be used to lead to the optimum choice of combination are:

a) The amount of Real Revenues, which are the revenues that an operator will gain from the hire of capacity to the customers of each combination.

b) The calculation of additional expected future profits for the satellite operator taking into consideration the standard deviation of the prices and consequently the corresponding risk.

Each combination has different maximum requested time of hiring the capacity. Therefore in order to be able to properly compare the scenarios it is necessary to reduce both of them to the same period of time i.e. at the same maximum month of hiring, and then to calculate the additional possible income that we can acquire from this 
left over free capacity that is called 'Remaining Capacity' (C Remaining).It is also possible that at specific months not all the available capacity of the transponder of the satellite will be occupied with each combination. This leads to the undesirable fact of not having maximum occupancy of the transponder of the satellite in each month. So the satellite operator could probably hire this available capacity to another possible future customer that is not included to the combination and gain more revenues. This is called 'Empty Capacity' ( $C$ Empty)

Let us examine the following example of two cases that a satellite operator would like to compare and decide which one leads to maximum revenues. These are Scenario 1 which includes the combination of the customer number 1, 2 and 3 (Figure 2) and Scenario 2 which includes the combination of the customer number 4, 5 and 6 (Figure 3) with the characteristics shown in Table 1.

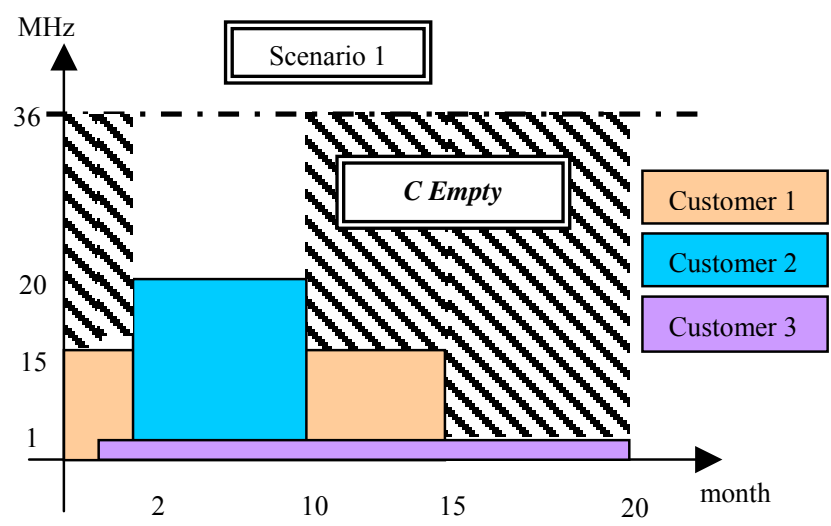

Figure 2: Scenario 1

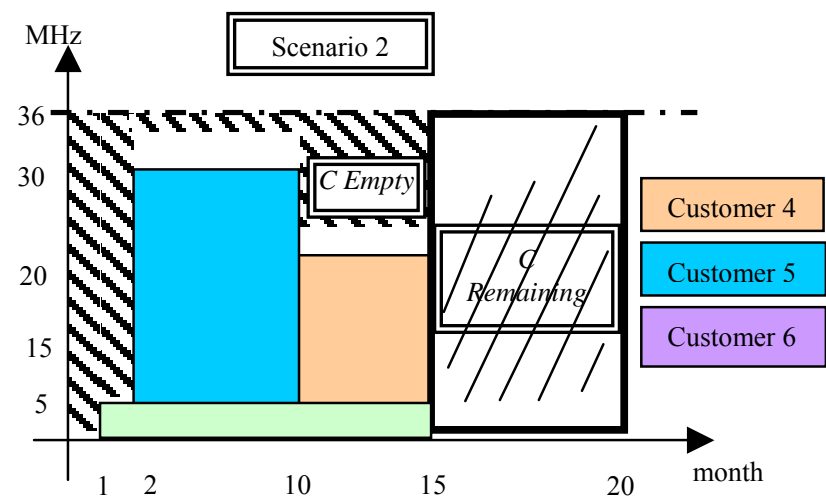

Figure 3: Scenario 2

In Scenario 1 the maximum duration of occupying the transponder is the 20th month, while in Scenario 2 is the 15 th month. The additional possible income that can be acquired from this left over free capacity is calculated. This is called 'Remaining Capacity' ( $C$ Remaining) and appears at the erased region in Figure 3.

Each Scenario does not lead to maximum occupancy of the transponder of the satellite in each month. This Empty Capacity (C Empty) is shown in the shaded region of
Figure 2 and Figure 3. For instance in Scenario 1 (Figure 2) there are $21 \mathrm{MHz}$ empty at the 1 st month, $20 \mathrm{MHz}$ empty at the $2^{\text {nd }}$ month, $0 \mathrm{MHz}$ empty at the 3 rd month (white space in Figure 2) etc. These expected profits are included to our calculations.

\section{Calculation of revenues from C Remaining}

For each scenario the possible revenues from the potential leasing of the Remaining Capacity are calculated. This calculation of the theoretical potential revenues is categorized depending on the type of service. Therefore the decision tree as shows in Figure 4 arises for each scenario.

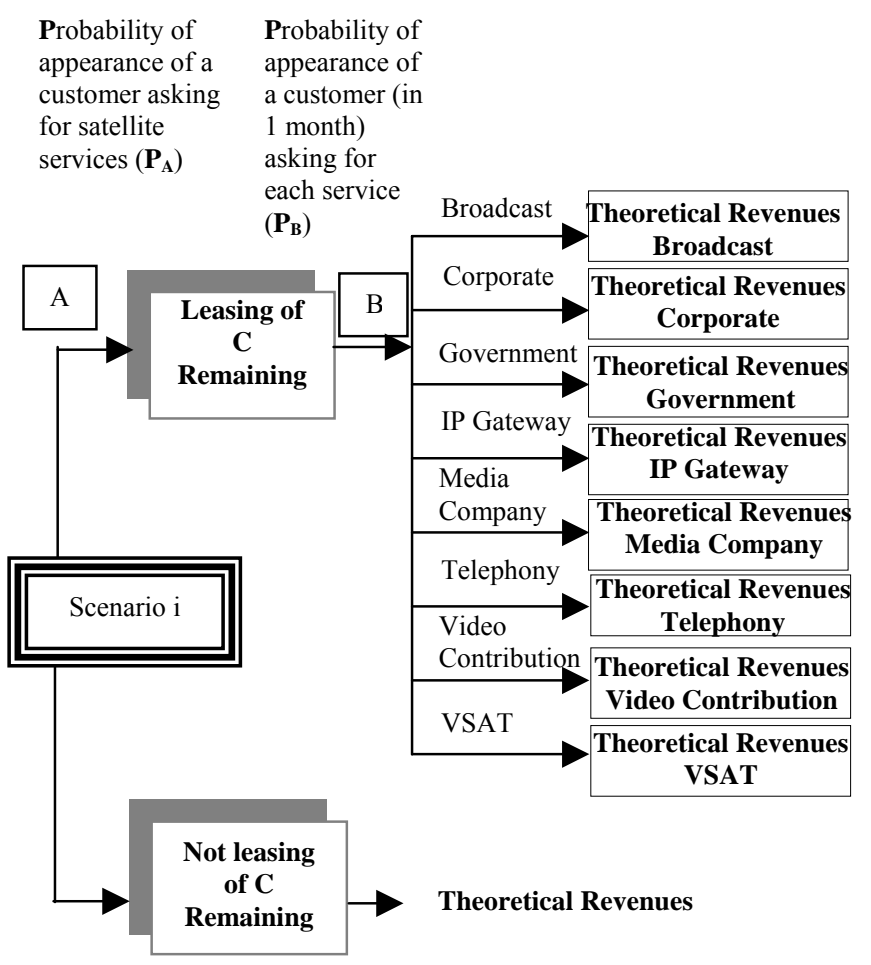

Figure 4: Revenues from C Remaining

The Expected Values of theoretical income for the Remaining Capacity depending on the type of service are being calculated as:

Probability of appearance of a customer (in 1 month) asking for each service $\left(\boldsymbol{P}_{\boldsymbol{B}}\right)^{*}$

*Mean value Price/36 MHz/month*

*Number of months*

*Probability of appearance of a customer asking for satellite services $\left(\boldsymbol{P}_{A}\right)$

The probable revenues for each service which are called Theoretical Revenues are calculated and categorized for each service as:

\section{Mean value Price/36 MHz/month* *Number of months}

The Expected Values of revenues is an intermediate calculated volume that is used only for comparison 
purposes of the Scenarios enclosing the corresponding risk while the size Theoretical Revenues is the real amount of money in Euros that can be acquired following each branch of the decision tree.

The Mean value Price per $36 \mathrm{MHz}$ per month is the mean value of pricing for each service referring to bandwidth of $36 \mathrm{MHz}$ that has been statistically calculated.

The standard deviation for the Expected Values and the Theoretical Revenues for each service are also calculated in order to have an estimation of the risk involved.

All the calculations of probabilities as well as the mean values of the prices of lease and standard deviations of prices are based on real data of the international market.

\section{Calculation of revenues from C Empty}

We proceed analogously calculating all possible revenues (Expected Values and Theoretical Revenues) that can result from the leasing of the 'Empty Capacity' up to the $36 \mathrm{MHz}$.

This calculation is categorized depending on the amount of bandwidth that is not used each month by the customers of each combination. This 'Empty Capacity' could potentially being hired and result to revenues.

The selected ranges of capacity in $\mathrm{MHz}$ is shown in
Figure 5 along with the corresponding probability of appearance of a customer in 1 month asking for each BW range and has been statistically computed by the available gathered data. A decision tree arises for each scenario.

The Expected Values of theoretical income for the 'Empty Capacity', depending on the not-leased bandwidth each time period, are being calculated as:

Probability of appearance of a customer (in 1 month) asking for each bandwidth $\left(\boldsymbol{P}_{c}\right) *$

*Mean value Price/MHz/month *

*number of $\mathrm{MHz}$ of Empty $\mathrm{C}$ * *number of months *

*Probability of appearance of a customer asking for satellite services $\left(\boldsymbol{P}_{\mathrm{A}}\right)$

While the Theoretical Revenues depending on the number of $\mathrm{MHz}$ that are not used are calculated as:

\section{Mean value Price/MHz/month * *number of $\mathrm{MHz}$ of Empty $\mathrm{C}$ * *number of months}

The standard deviation for the Expected Values and the Theoretical Revenues for each bandwidth are also calculated.

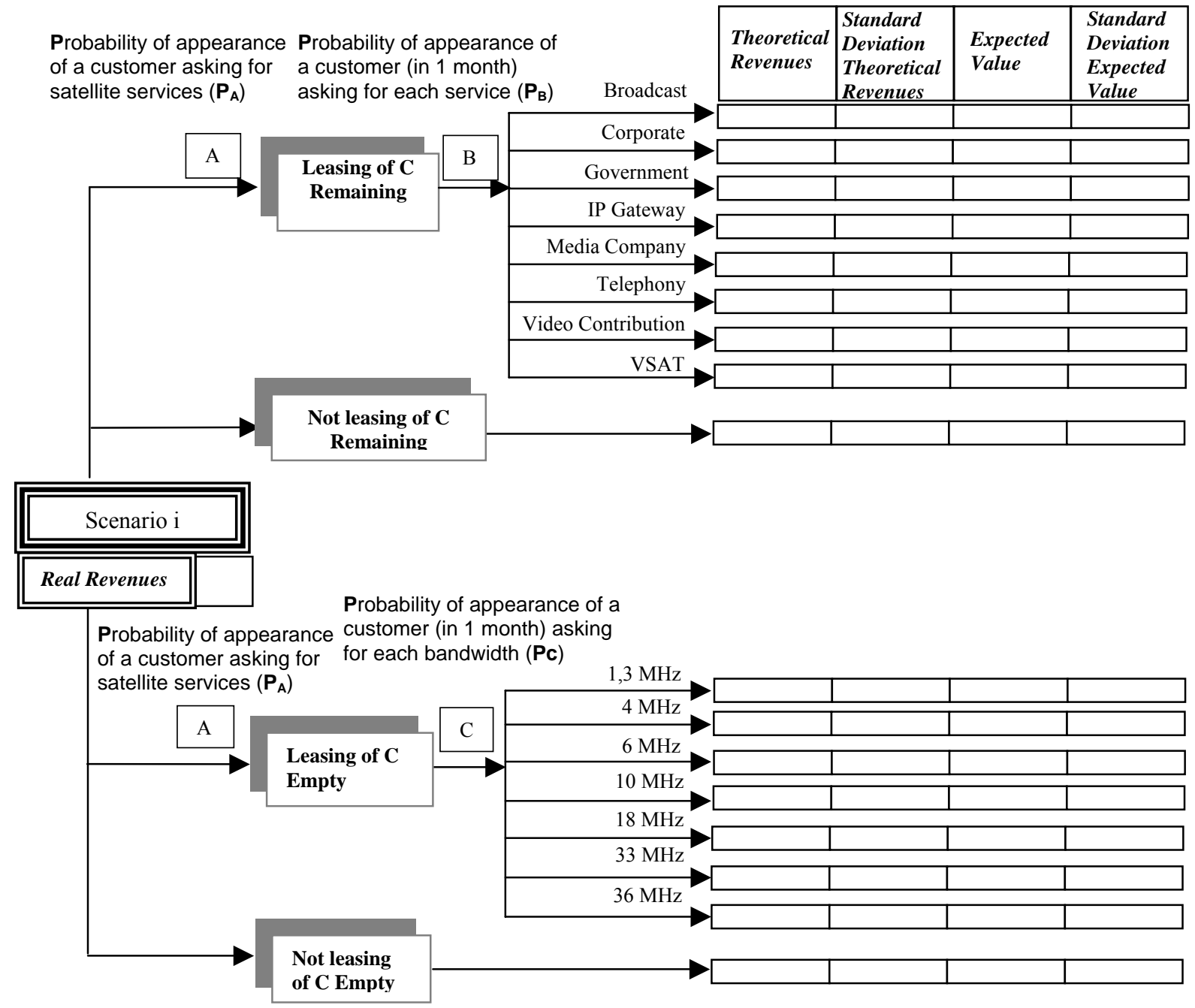

Figure 5: Output of the model Decision Tree 


\section{CONCLUSIONS}

All of these evaluated data are presented in the form of a unified decision tree (Figure 5), which is the output of the model.

The amount of Total Expected Revenues that will estimate the optimum policy for the firm is the sum of all potential revenues. This sum consists of the Real Revenues, plus the Expected Value resulting from the leasing of the Empty Capacity plus the Expected Value resulting from the leasing of the Remaining Capacity including their standard deviations. The Total Expected Revenues gives a range of values, defining the best and worst case scenario for the revenues of the satellite operator.

Total Expected Revenues $=$

Real Revenues+

(Expected Value from C Empty \pm

Standard Deviation Expected Value C Empty) +

(Expected Value Revenues from $C$ Remaining \pm

Standard Deviation Expected Value C Remaining)

The real amount of money that will possibly result to the satellite operator is:

\section{Total Revenues $=$}

Real Revenues+

(Theoretical Revenues from C Empty \pm

Standard Deviation Theoretical Revenues $C$ Empty) +

(Theoretical Revenues from $C$ Remaining \pm

Standard Deviation Theoretical Revenues $C$ Remaining)

This gives the opportunity to the manager of the satellite provider to determine which is the most profitable combination now and in the future. The decision is made using the amount of Total Expected Revenues. This is not the real revenue that can be acquired, but it is an intermediate amount, taking into account corresponding probabilities, used for comparison of the scenarios.

In order to make the decision, we look first for the highest Real Revenues and then the possible revenues that can result with their corresponding standard deviation. This results to a range of Expected Revenues with central value: Real Revenues +Expected Value from C Empty +Expected Value Revenues from C Remaining,

upper limit: Real Revenues +Expected Value from $C$ Empty +Expected Value Revenues from $C$ Remaining +Standard Deviation Expected Value $C$ Empty + Standard Deviation Expected Value $C$ Remaining,

and lower limit: Real Revenues +Expected Value from $C$ Empty +Expected Value Revenues from C Remaining Standard Deviation Expected Value C Empty - Standard Deviation Expected Value $C$ Remaining.

This final decision depends on the extent of risk the firm is willing to take and the certain policy that wants to apply. For a risk loving decision maker the policy with the upper limit of Total Expected Revenues is chosen, whereas for a risk averse decision maker the policy with the lower limit of Total Expected Revenues is chosen. The real amount of money that will result from each decision is the amount of Total Revenues of the corresponding scenario.

The usefulness of the model and analysis presented here for any satellite operator is clear. They could also be useful to related types of activities where leasing for specific volumes to customers is the essence of the business enterprise.

Finally let us note that a more extended version of the work presented here with all the mathematical details (discrete time stochastic dynamic programming formulation and solution) will be presented in future publications and will also be available in the forthcoming Phd Thesis of the first author, together with the related code.

\section{REFERENCES}

Bertsekas, D.P. "Dynamic Programming and Optimal Control" vol. I, 3rd Edition 2005 and vol. II, 2nd Edition 2001, Athena Scientific, Belmont, MA

Guikema S.D. and Pate'-Cornell, M.E. 2002. "Component choice for managing risk in engineered systems with generalized risk/cost functions" ELSEVIER Reliability Engineering and System Safety, 227-238

Ross, S.M. 1983. "Introduction to Stochastic Dynamic Programming" Academic Press.

Schouwenaar, M. and Martin, E. 2003. "Optimization of a Telecommunications Billing System" Proceedings of the 2003 Winter Simulation Conference, 1843-1847

ELENA SARRI is a Phd candidate at the Dept. of Electrical and Computer Engineering National Technical University of Athens, Greece. She holds a BSc in Physics (4 year program) and a 2 year Mphil degree in Electronics and Radioelectrology, from the Depts. of Physics and Informatics all at the National Capodistrian University of Athens. She is a Laboratory Instructor at the Technological Education Institute of Piraeus, Dept. of Electronic Engineering. Before starting her Phd, she worked as a Telecommunication Engineer at the Department of Design and Development of Networks and New Technologies at a Local Mobile Provider. Her current research interests are in the area of Techno-Economic analysis and evaluation of Telecommunication systems as well as in Optimization and Decision Making under uncertainty. Her e-mail address is: elena@netmode.ntua.gr.

GEORGE. P. PAPAVASSILOPOULOS received the Diploma in Mechanical and Electrical Engineering from the National Technical University of Athens in 1975 and the MSc and $\mathrm{PhD}$ degrees in Electrical Engineering from the University of Illinois at Urbana Champaign in 1977 and 1979 respectively. In 1979 he joined the Dept. of Electrical Engineering-Systems of the University of Southern California as an Assistant Professor and was later promoted to Associate and Full Professor with tenure. In 2000 he joined the Dept. of Electrical and Computer Engineering of the National Technical University of Athens as Full Professor. His main research interests are in Game Theory, Control and Optimization Algorithms. He is also interested in applications in Networks, Energy Policy, Economics and Bioengineering. He has conducted research on several theoretical and applied problems dealing with game theory, optimization algorithms, parallel and distributed computing, and nonlinear control. This research has been funded in the past by NSF,AFOSR,NASA(JPL) and is currently funded by Greek research grants. His e-mail address is: yorgos@netmode.ntua.gr. 